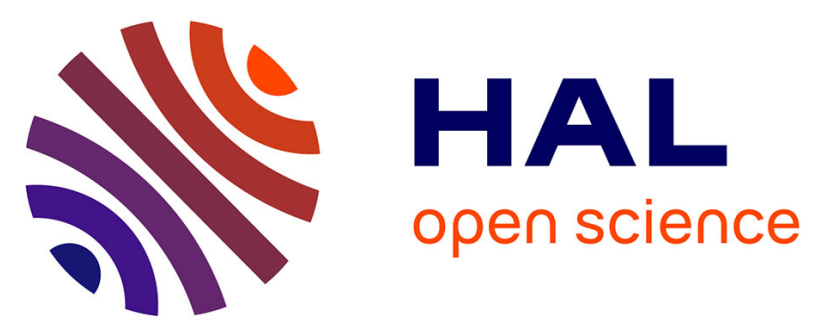

\title{
Guiding the training of users with a pattern similarity biofeedback to improve the performance of myoelectric pattern recognition.
}

Étienne de Montalivet, Kevin Bailly, Amélie Touillet, Noël Martinet, Jean Paysant, Nathanael Jarrasse

\section{To cite this version:}

Étienne de Montalivet, Kevin Bailly, Amélie Touillet, Noël Martinet, Jean Paysant, et al.. Guiding the training of users with a pattern similarity biofeedback to improve the performance of myoelectric pattern recognition.. IEEE Transactions on Neural Systems and Rehabilitation Engineering, inPress, pp.1-11. 10.1109/TNSRE.2020.3003077 . hal-02884543

\section{HAL Id: hal-02884543 \\ https://hal.science/hal-02884543}

Submitted on 29 Jun 2020

HAL is a multi-disciplinary open access archive for the deposit and dissemination of scientific research documents, whether they are published or not. The documents may come from teaching and research institutions in France or abroad, or from public or private research centers.
L'archive ouverte pluridisciplinaire HAL, est destinée au dépôt et à la diffusion de documents scientifiques de niveau recherche, publiés ou non, émanant des établissements d'enseignement et de recherche français ou étrangers, des laboratoires publics ou privés. 


\title{
Guiding the training of users with a pattern similarity biofeedback to improve the performance of myoelectric pattern recognition.
}

\author{
Etienne de Montalivet ${ }^{1}$, Kevin Bailly ${ }^{1}$, Amélie Touillet ${ }^{2}$, Noël Martinet ${ }^{2}$, Jean Paysant ${ }^{2}$ and Nathanaël Jarrassé ${ }^{1}$
}

\begin{abstract}
Next generation prosthetics will rely massively on myoelectric "Pattern Recognition" (PR) based control approaches, to improve their users' dexterity. One major identified factor of successful functioning of these approaches lies in the training of amputees and in their understanding of how those prosthetics works. We thus propose here an intuitive pattern similarity biofeedback which can be easily used to train amputees and allow them to optimize their muscular contractions to improve their control performance. Experiments were conducted on twenty able-bodied participants and one transradial amputee. Their performance in controlling an interface through a myoelectric PR algorithm was evaluated; before and after a short automatic user training session consisting in using the proposed visual biofeedback for ten participants, and using a generic PR algorithm output feedback for the others ten. Participants who were trained with the proposed biofeedback increased their classification score for the retrained gesture (by $39.4 \%$ ), without affecting the overall classification performance (which progressed by $10.2 \%$ ) through over-training and increase of False Positive rate as observed in the control group. Additional analysis indicates a clear change in contraction strategy only in the group who used the proposed biofeedback. These preliminary results highlight the potential of this method which does not focus so much on over-optimizing the pattern recognition algorithm or on physically training the users, but on providing them simple and intuitive information to adapt or change their motor strategies to solve some misclassification issues.
\end{abstract}

\section{INTRODUCTION}

Since the early seventies, the standard way for upper-limb amputees to control a powered active prosthesis is based on the use of myoelectric signals measured over the stump as control inputs. Such approach, initially developed in the late sixties in the U.S.S.R. [1], simply relies on the use of ElectroMyoGraphic signals (EMG) from two antagonistic muscles of the residual limb. Often, each active prosthetic joint that composes the substituting limb is sequentially controlled by the same control inputs. So, despite the potential possibilities offered by the new biomimetic prostheses like

This study was supported by the ANR (project PhantoMovCOntrol ANR15-CE19-0008-02) and the Labex SMART (ANR-11-LABX-65) supported by French state funds managed by the ANR within the Investissements d'Avenir programme under reference ANR-11-IDEX-0004-02.

${ }^{1}$ E. de Montalivet, K. Bailly and N. Jarrassé are with the CNRS, UMR 7222, ISIR / INSERM, U1150 Agathe-ISIR, Sorbonne Université, Paris, France [firstname.lastname] at sorbonne-universite.fr

${ }^{2}$ A. Touillet, N. Martinet and J. Paysant are with the Centre Louis Pierquin, Institut Régional de Médecine Physique et de Réadaptation, UGECAM Nord-Est, Nancy, France [firstname. lastname] at ugecam.assurance-maladie.fr whole robotic arms [2] or polydigital hands [3], their control remains complex, as it is far from intuitive, and offers few functional Degrees of Freedom (DoF) [4].

\section{A. Pattern recognition (PR) techniques}

To overcome these limitations, pattern-recognition (PR) approaches have been developed since the late 60s/70s [5], [6], [7] aiming a more precise decoding of myoelectric signals in order to improve the recognition of different muscle activation patterns and thus to control more types of movements. This requires the use of multiple recording sites, a precise extraction of signal characteristics and a multidimensional classification architecture relying on machine learning techniques. While well established and extensively studied in the context of research, such approaches have hardly been applied to commercially available prostheses.

Indeed, although successful in research facilities (with classification rates over $90 \%$ reported in the literature [8], [9] for sets of more than 10 different movements), the current limited robustness of those PR techniques remains a major obstacle to every day use by amputees. Misclassification of myoelectric patterns can come from the user (variability in muscular contractions because of effort modulation, motor intention variations or muscle fatigue) but also from numerous external factors like the changes in the stump posture, the movements of electrodes or the sweat and variations of skin impedance [10]. These factors will affect the sEMG signals and thus decrease the successful classification rate and lead to user dissatisfaction.

Yet numerous solutions are actually developed that could compensate for the listed issues. Examples of these solutions are (1) robustness to electrodes shift [11], [12], (2) use of osseointegration [13] to eliminate the issues due to the socket, (3) electrode implantation [14] minimizing the issue with skin impedance and movements, and of course (4) more robust architectures of $\mathrm{PR}$, integrating the stump posture (tracked through IMUs) to integrate the actual arm posture in the signal classification [15].

\section{B. Training of users and algorithms}

Another major factor of successful recognition of sEMG signals lies in the training of amputees and in their understanding of the functioning of prosthesis control algorithms. Indeed, myoelectric control, especially PR, principally relies on the consistency of muscular patterns, and these are complex to obtain for two reasons. First, we can contract 
our muscles in numerous different ways to perform a similar gesture (thanks to muscle redundancy [16]) and second, we have a limited sensory feedback concerning our muscle contractions. Additionally, this last effect is worse with an amputated limb that suffered from a neuromuscular reorganization and does not offer any visual feedback of the movement generated by the muscular contractions. Thus, even for conventional myoelectric control schemes, training of users and algorithms have been intensively studied within the last years, with the development of numerous dedicated approaches and tools such as virtual environments ([17], [18]) or effective mobile games [19] for myoelectric control training, dedicated systems for the training of precise control of muscular contraction amplitudes [20], or visual biofeedback to train user's in regulating their grasping force and stabilize their muscle contractions [21], among other numerous research works.

Similarly, for the specific case of machine-learning based control (such as PR or regression), several recent studies have been focusing on the training of participants control abilities, in addition to adaptive algorithms able to adapt to the variations of subject's patterns across training sessions. Indeed, as shown in a study investigating the changes in EMG classification performance over 11 consecutive days, the performance of the motor tasks is consistent over practicing time, resulting in more repeatable EMG patterns than at initial use, even when no performance feedback was used [22]. Hahne et al. [23] also verified that man-machine coadaptation (closed-loop real-time learning schemes in which both the user and the machine learn simultaneously to follow a common target) was helpful to improve a regression-based myoelectric control. Scheme et al. [24] were able to improve PR performance by training the users to modulate their myoelectric contraction amplitude as required by generic proportional control. Powell et al. [25] studied the effect of training sessions with visual feedback and training guided by a therapist on transradial amputees and found that the control was improved after two weeks of training with a virtual prosthesis. Nonetheless, their protocol required an important implication of the experimenter who, during dedicated sessions and based on the PR confusion matrices analysis, guided the amputated participants to modify their gesture accordingly and to iteratively refine each subject's muscle contraction during training. On the contrary, a recent study [26] on 37 able-bodied population showed that the effects of training were the same, whether visual feedback or experimenter coaching were used or not during training. Whatever the nature of training, it is clearly becoming a mandatory step for amputees fitted with prosthetics.

Since providing a visual feedback on the PR algorithm's raw output may not be sufficient to guide the adaptation of subject motor behavior, several teams developed dedicated feedbacks to assist users during training protocols. For example in [27], a polar plot of EMG amplitude as a function of electrode location was used to assist one transradial amputee in improving his control performance through training. In [28] an innovative classifier-feedback- based user training strategy is used to provide real-time "clustering-feedback" (i.e. 2D projected representation of online EMG signal input as well as the centroids of the training samples) to help subject's adjusting their muscular contraction strategy. Yet, very little research has been conducted on developing a visual biofeedback to guide, during the PR training phase, the users in modulating their way of performing a phantom limb gesture (i.e. exhibiting a muscular contraction pattern) with the aim of maximizing the differences between contraction patterns and minimizing similarities between movement classes and thus ease the PR algorithm performance.

In this study we thus propose, based on a generic PR architecture, an intuitive visual feedback providing -in real-timeinformation on the distinguishability of the contraction pattern according to the other to be used as control inputs, along with some feedback on the muscular contraction level (to encourage users in regulating their effort). An experimental campaign was conducted on twenty able-bodied participants and one transradial amputee. Their training session consisted in using the proposed intuitive visual biofeedback for one (experimental) group and using a standard PR algorithm output feedback for the other (control) group. Before and after this training session, their performance in controlling an interface through an automatic PR algorithm (i.e. without any skilled experimenter guidance) was evaluated. In order to evaluate the potential of the proposed approach, differences in the PR algorithm performances before and after the training session were analyzed through different metrics.

\section{PRINCIPLE}

We first want to address a critical issue of the current PR training protocols, that require users to perform discrete generic gestures (like "closing the hand") to be used as "references". Those gestures generally have a large intravariability and can be performed in numerous different kinematic way. This results in numerous different muscular contraction patterns, that are still labeled by the same name for the PR algorithm. What we propose here is a visual biofeedback on the muscle contraction patterns from a machine's (here a PR algorithm) point of view, which can be used by users to explore their muscular strategies, to find more efficient and robust strategies, and then, once identified, to update the training of the PR algorithm accordingly.

While such approach could be used with numerous type of PR architecture, we chose in this study to use a linear discriminant analysis (LDA) classifier [29] which is commonly used for myoelectric pattern recognition.

\section{A. Concept}

It is generally possible to improve the classification performance of physiological signals, by involving the participant in more or less guided training and exploration steps:

- Step 1: PR training $\rightarrow$ PR testing

- Step 2: Subject training/exploration (through practice, guided or not) 
- Step 3: PR training update $\rightarrow P R$ testing

- Step 4: Repetition of Steps $2 \& 3$ (until performance improves)

Such steps can then be repeated several times, across several sessions and days, with the aim of improving the overall performance.

The generic method, such as that used in [25], trains the participants (by allowing them to explore strategies) in Step 2 with the discrete output (i.e., the label of the recognized class) of the classifier (created in Step 1) as a feedback. The subject should then understand which movements lead to the right classification. Such raw output feedback (RF) helps the subject to improve its repeatability. The creation of the training set in Step 1 is thus decisive since the improvement of the classifier's performance will rely on the precise reproduction of those initial movement demonstrations. We hypothesize that, if a participant generates confusing training samples labelled as class $C_{1}$ in a three classes set $\left\{C_{1}, C_{2}, C_{3}\right\}$ as illustrated in Fig 1 . Step 2 will help the subject minimizing the variability in his movement repetition which will generate a convergence of the data towards the associated class centroid $\mathbf{m}_{1}$ of the cluster of data associated to class $C_{1}$. If the participant do not attend to explore by himself -even accidentally- novel strategies, or is guided to do so, after retraining, new cluster $\mathcal{C}_{1 R F}$ variability should decrease but its centroid $m_{1 R F}$ would remain the same. We also believe that with Pattern Similarity biofeedback (PSB), a participant could be trained to change his/her way to exhibit an unwellclassified movement (identified at the end of step 1 during the PR testing phase). During the training/exploration of Step 2, instead of the discrete raw output of the PR algorithm, such continuous biofeedback would inform the subject of the similarity of performed gesture with the others of the training set that were built in Step 1, from a classification point of view. With such a method, we expect that the training will lead to a displacement of the centroid of the cluster associated to the retrained movement, far from other centroids. More explanation on cluster and centroid definition in LDA can be found in [30].

Such a method should help the participant to find new ways of performing a muscle contraction for a given class in light of the other contractions patterns to be used, from the machine's point of view. Considering the classification of 3 movements $\left\{M_{1}, M_{2}, M_{3}\right\}$ with therefore a three classes set $\left\{C_{1}, C_{2}, C_{3}\right\}$ with their associated centroids $\left\{m_{1}, m_{2}, m_{3}\right\}$, we hypothesize here that, if a participant has an unwellclassified movement (defined by cluster $C_{1}$ and centroid $m_{1}$ in Fig 1), the user training (Step 2) will help him/her to find a way of performing the given gesture that moves the centroid of the new cluster $\mathcal{C}_{1 O B}$ away from the other class centroids $m_{2}$ and $m_{3}$. Such biofeedback method takes advantage of the fact that a class of movements can be achieved through several strategies: there is indeed no unique physiological way of closing a hand, opening a pinch, or turning a wrist. Similarly, for amputees, there is generally not only a single way of performing one given "phantom movement" type. Thanks to this feedback, users could, for example, test sev- eral ways of performing a gesture of one class and determine the one that makes the associated contraction patterns very different from the gestures of the other classes, with distance being measured from the machine's point of view (i.e. in the projected multidimensional space of data features extracted from sEMG signals).

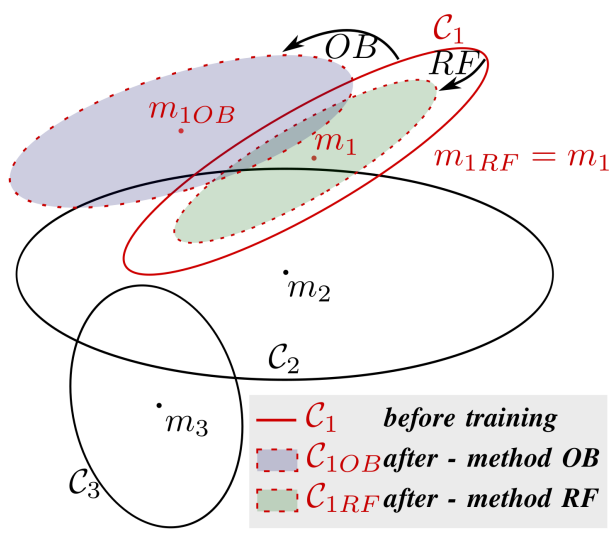

Fig. 1: Hypothetical effect of training with raw output feedback (RF) and pattern similarity biofeedback (PSB). Clusters represent multidimensional ensembles of data (features) associated to the movement classes projected onto a 2D subspace (i.e., the training space created at step 1). $C_{1}$ has been retrained with the two methods (resulting in $C_{1 R F}$ and $C_{1 O B}$ ).

The proposed protocol of use of such tool is as follows: the final testing of step "1)" (as listed in Section II-A) is used to determine the problematic class (the one with the lowest classification scores); then the subject is asked, during training step "2)”, with help of guidance provided by the pattern similarity biofeedback, to find a way of performing the movement of the problematic class that is less confusing for the PR algorithm. Once the user has determined a more efficient ways to perform their gesture, the classification training data for the given movement are updated, with this new set of data replacing the one of the previously confusing sets. Such steps can then be repeated to improve other problematic classes.

\section{B. Pattern Similarity biofeedback design}

While it has been showed that discrete feedback during training improves classification [31], we expect that providing, a biofeedback -during the exploration stage- informing the subject in a continuous and quantified way on the similarity of the achieved movement with movements of the other classes, will have beneficial effects. To this aim, rather than providing dense information on the different muscle contraction amplitudes like in [27], we designed a simplified index showing the similarity of a given muscular contraction pattern with the other movement classes, and this, from the point of view of the PR algorithm. Concretely, we compute the distance between the point representing a given performed gesture (of the class to be retrained) to the centroids of the other classes in a previously constructed 
projected subspace. The majority of pattern recognition algorithms used for prosthetic control exploit numerous different features (characterizing signal aspects such as its frequency, complexity, etc.) in addition to the RMS value of the EMG channels. We therefore decided to build a dedicated visual feedback providing more representative information (i.e. similar to what the classifier really analyses) rather than just providing EMG RMS radar plots as it has been done in some other studies.

For each class, except the current retrained one, the distance $r_{i}$ in the projected training space between the current posture and all the centroids of the classes is computed:

$$
\forall i \in[1 ; c] \backslash c_{r}, r_{i}=\sqrt{\sum_{j=1}^{(c-1)}\left(x_{j}-m_{i j}\right)^{2}}
$$

Where $c_{r}$ is the current retrained class, $\mathbf{x}$ is the current sample from the sensor projected in training space, $\mathbf{m}_{i}$ the centroid of class $i$. All the distances are represented on a $c-1$ radar plot. On each branch is plotted the $i^{t h}$ centroid $c_{i}$ at the distance $r_{i}$ from the center. The center represents the current sample which is the current explored posture projected in the training space. In order to reduce the dimensionality of $\mathbf{r}$ and make the feedback more intuitive and simple to understand (especially in real-time) for the participant, a circle is plotted in real-time with radius $R=\min r_{i}, i \in[1 ; c] \backslash c_{r}$.

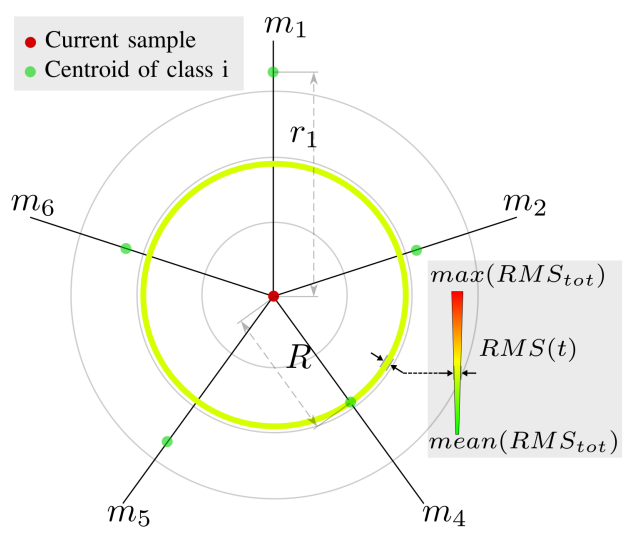

Fig. 2: Pattern similarity biofeedback illustration for a 6classes classifier. Class 3 is here retrained.

During the exploration stage, the class to be retrained is not taking into account because a new better way of performing the gesture associated to this class is to be found. The circle is continuously changing size, depending on how far from centroids (of other gestures) the current posture is. Because the rest posture is often very-well classified, the circle should almost be a point when subject is not moving. Then, exploring a new posture will increase its diameter as much as the explored posture's projected sample is far from the projected centroids: the bigger the circle is, the further the closest centroid is in the projected training space. In order to avoid strategies using stronger muscle contractions to differentiate one gesture from the others (which would lead to more muscular fatigue) a pure RMS-based feedback is also used. Based on the RMS values of the first training set, average and max values are computed and used to modulate in real-time the circle's color accordingly, from green for no contraction, to red indicating that the maximum RMS value (measured during the first training) is reached, as shown in Fig. 2.

\section{Material AND Methods}

In order to validate the efficiency of the proposed biofeedback compared to state-of-the art training approaches, two tests with a similar setup were performed: one preliminary on twenty able-bodied participants and one on a transradial amputated participant.

\section{A. Experimental setup}

Measurement of the surface myoelectric activity of the forearm is done by a dedicated 8 channel electrophysiological signal-recording system (Myoband (c) from Thalmic Labs) with an 8-bit resolution. No specific skin preparation was used before placing the armband on the participants. Armband was placed on the forearm at approximately $5 \mathrm{~cm}$ from the elbow joint (as shown on Fig. 3) left.

The PR algorithm is executed on a Raspberry Pi 3 running on Jessie OS (Linux Debian). The real-time program (coded in $\mathrm{C}$ ) reads the armband data through a Bluetooth communication and processes the classification. The sEMG are acquired at a $200 \mathrm{~Hz}$ frequency, while the features are computed from the sEMG using a 256-ms-sliding analysis window with a 64-ms-overlap between successive windows. The root mean square value [32], the first 4 autoregressive coefficients [33], [34], the zero crossing and the wave lenght of the sEMG were extracted from each channel and used to create the feature vector (which dimension is here equal to 56). Output data are broadcast through wifi to the graphical user interface.

The Graphical User Interface (GUI) is running on Matlab. For the steps 1 and 3, the interface only provides readable instructions to the participant (i.e. the current movement to achieve or rest). For the user training phase (step 2), depending on the participant group, a specific feedback was displayed.

\section{B. Participants}

Twenty naive able-bodied participants (in the 21-68 age range) were recruited among the laboratory members. One amputated participant with unilateral transradial amputation of traumatic origin was selected to participate to the study. This study was carried out in accordance with the recommendations of the Université Paris Descartes ethic committee CERES ( $\mathrm{N}^{\circ}$ IRB 20151900001072), which had approved the protocol. All participants provided written informed consent to participate in the study, and the patient gave written permission for publication of photographs for scientific and educational purposes. The protocol was performed in accordance with the Declaration of Helsinki.

A 26 year old male participant with a traumatic right forearm amputation (2/3 of the forearm left) who had a past 
experience of myoelectric control (use, on a daily basis, of a myoelectric prosthesis composed of an active wrist rotator and a polydigital hand) was also recruited to preliminary assess the effects of the proposed approach on a more realistic case. The amputated participant was followed-up at the Louis Pierquin Centre of the Regional Institute of Rehabilitation, Nancy, France.

\section{Experimental protocol}

Two distinct experimental campaigns were conducted: a first one on able-bodied participants to evaluate the effects of the pattern similarity biofeedback (PSB) compared to a control group training with a raw output feedback (RF), a second one on the amputated participant to validate the observed effect in a more realistic scenario. All experiments followed the protocol presented in Section II-A

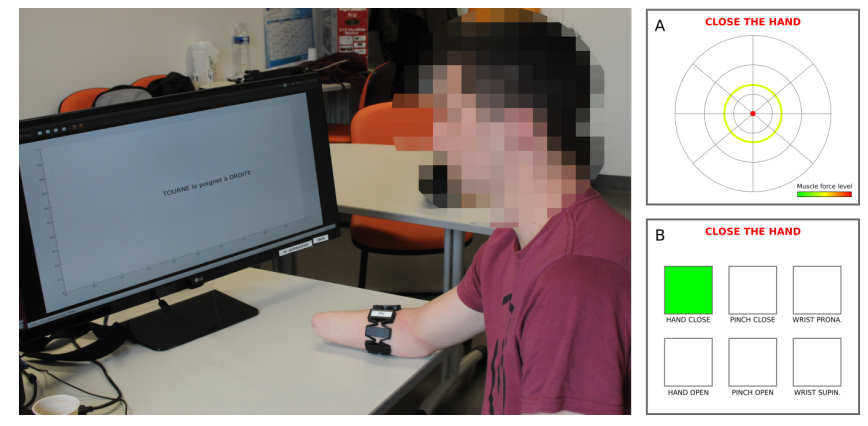

Fig. 3: Left: amputated participant equipped with the MyoBand during the experiment. Right: View of the two different graphical interfaces used during the experiments. Top: Pattern Similarity Biofeedback GUI used with Group A (PSB-group) and amputated participant. Bottom: Raw-output feedback GUI used with Group B (RF-group).

1) Preliminary experiment with able-bodied participants: The twenty able-bodied participants were randomly assigned to two groups of the same size: Group A (PSB-group) attends a user training (step 2) with a Pattern Similarity Biofeedback and Group B (RF-group) attends user training (step 2) with a Raw-output Feedback. Preparation and both steps 1 and 3 (as defined in II-A) were identical for the two groups. The participants were seated on a chair with their arm placed along the body. They faced the screen to read instructions on the graphical interface. Once the armband was placed over their right forearm, participants kept it during all the experiment without removing it. Rest were first recorded to compute thresholds used to automatically cut off labeled records.

The following protocols were followed:

Step 1 consisted for participants in executing the instructions. It lasted approximately 3 minutes. 5 repetitions for each of the 6 chosen movements were randomly displayed, each execution lasting 3 seconds, following by 3 seconds of rest. The seven movements to classify were the Wrist Pronation $(W P)$ and Supination (WS), the Hand Closing $(H C)$ and Opening $(H C)$ and the Pinch Closing $(P C)$ and Opening $(P O)$ and the rest. The choice of having a specific pinch opening class, different from the whole hand opening one, was motivated by a practical observation. Indeed, in present polydigital prosthetic hands, there exists a common and largely used "quick grip" (hand posture mode) for precise manipulation which place the 3 last fingers closed and leaves only the thumb and index active (opening and closing) to perform a precise pinch (i.e. the "Standard precision pinch closed" in iLimb/Ossür ${ }^{\complement}$ Touch Bionics hands) without being bothered by the other fingers movements. Thus two separate opening classes were used. The LDA projected space was then created, and the PR testing started. Again, the participants were asked to perform the 30 randomly displayed postures. The confusion matrix were then plotted and used to determine the worst-classified class $c_{r}$ to be retrained.

Step 2 which lasted about 3 minutes, consisted in performing 30 repetitions of the movement of the class $c_{r}$ to retrain. Each group was provided with a different visual feedback, as shown in Fig. 3 right. For Group A, the graphical interface displayed the pattern similarity biofeedback described in IIB. Participants were asked to explore movements trying to maximize the size of the circle without turning it into red (i.e., without using excessive muscle force). For Group B, the graphical interface displayed a 6 boxes plot showing the raw output of the classifier. The color of the box of the detected class was modulated similarly to the feedback of Group A to provide information on the muscle contraction level. Participants were asked to explore movements while trying to activate the retraining movement corresponding box on the plot without turning it into red.

Step 3 was the exact reproduction of Step 1 (same duration), except that participants were asked to perform the movement of the class $c_{r}$ in the optimal way which was discovered during Step 2. The entire protocol lasted approximately 10 minutes.

2) Experiment with amputated participant: One forearm amputee tested the protocol with the pattern similarity biofeedback only. The protocol was exactly the same as group A, except that he had 5 seconds to exhibit movements during the 30 random repetitions.

\section{Metrics}

Three metrics were used to assess the improvement allowed by the training with the two different feedbacks. The classification scores variations were computed for both groups considering all movement classes and the retrained movement class only. The False Positive rate variation was also analyzed. Performance variations for both groups were compared, also by computing the Bootstrapped CI (confidence intervals).

Data analysis was performed in Python 3 (Python Software Foundation, http://www.python.org using SciPy. The normality of data distribution was assessed by the ShapiroWilk test. Comparisons were made by using non-parametric test (Mann Whitney) for the sample data with non-normal distribution, and Student's $t$-tests for the other data. The level of significance was set at $p<0.05$. The class separability 


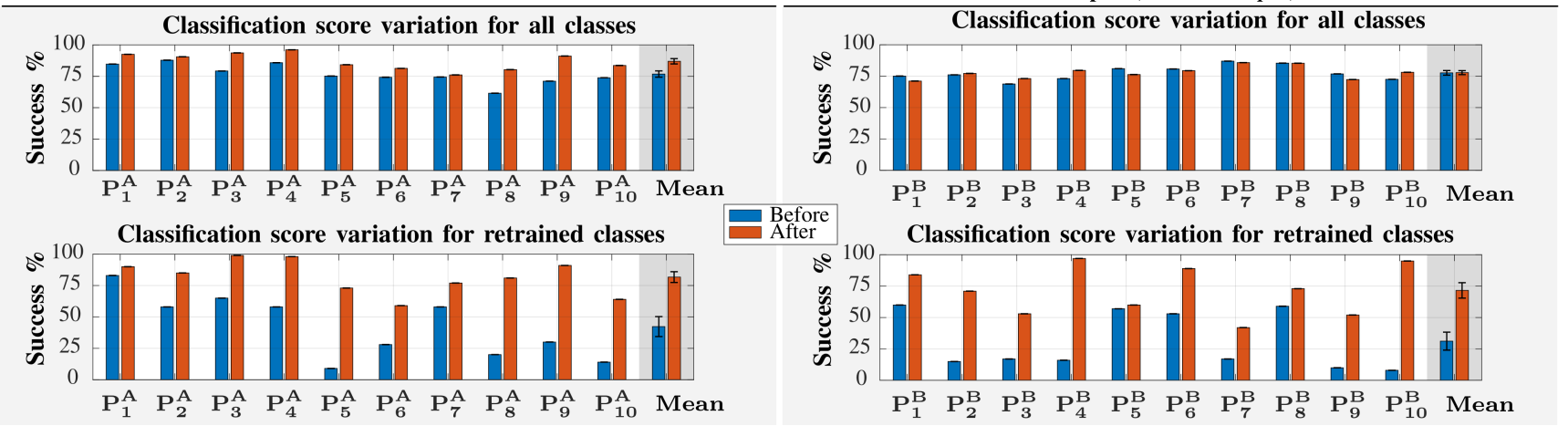

Fig. 4: First row: classification scores for all movement classes before (blue) and after (red) training with the Group A using pattern similarity biofeedback (Left) and the group B using raw PR output feedback (Right). Second Row: classification scores for the retrained class before and after training with the Group A (Left) and the group B (Right).

was assessed by computing $J_{3}$, an index based on scatter matrix (taken from [35]), defined as $J_{3}=\operatorname{trace}\left(S_{w}^{-1} \cdot S_{b}\right)$ where $S_{w}$ is the within-class scatter matrix and $S_{b}$ is the between-class scatter matrix. In addition, the variation of the overlapping in clusters distribution obtained after training was computed (see Appendix VII-A). Finally, some radar plots illustrating the average RMS contraction level on each sEMG channel associated to the class retrained in step 2 were generated to study the variation in muscle contraction strategies before and after the training of the participants.

\section{RESUlTS}

\section{A. Preliminary experiments on able-bodied participants}

1) Variation in classification performance after training: As shown in Figure 4 Group A started with a before-training average global classification score of $76.8 \%$ with a $95 \%$ (C.I.) of $[76.7,80.8]$ and Group B with an after-training average classification score of $77.6 \%$ with a $95 \%$ (C.I.) of $[77,80.6]$. Group A ended with an after-training average global classification score of $86.9 \%$ with a $95 \%$ (C.I.) of $[85,89]$ and Group B with an after-training average classification score of $77.8 \%$ with a $95 \%$ (C.I.) of $[76,79.8]$. Statistical difference is observed in performance between groups after training ( $p=0.003)$ but not before $(p=0.804)$, which indicates a clear effect of the feedback method.
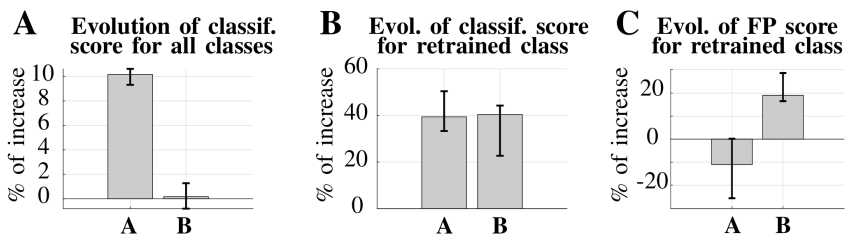

Fig. 5: Averaged classification score variation, for the two groups $\mathrm{A}$ and $\mathrm{B}$, after training with representation (as error bars) of the $95 \%$ Confidence Interval, for all movement classes (A) and for the retrained class only (B). Evolution of False Positive percentage for the retrained class is also shown $(\mathrm{C})$.

Figure 5 shows that, when considering all the movement's classes and all group participants, Group A (PSB-group) obtained an average increase in classification score of $10.2 \%$ with a $95 \%$ Confidence Interval (C.I.) of $[9.3,10.6]$ while the control Group B (RF-group) obtained a lower increase of $0.2 \%$ with a $95 \%$ (C.I.) of $[-0.8,1.3]$. Statistical difference is observed in global performance variation between groups after training for group A $(p=0.006)$ but not for group B $(p=0.945)$, which indicates a clear effect of the feedback method.

Looking only at the "retrained" class, Group A (PSBgroup) obtained a statistically significant $(p<0.001)$ average increase of the classification score of $39.4 \%$ with a $95 \%$ (C.I.) of $[33.3,50.4]$ while the control group B obtained an average increase of $40.4 \%$ (C.I.) of $[22.7,44.2]$ (with $p=0.002$ ). No statistical difference in performance between groups for the retrained class could be observed, whether before $(p=0.099)$ or after training ( $p=0.197)$, indicating no clear effect of the feedback method.

Observing the variation of the percentage of False Positive (FP) for the retrained class (cf. Figure 5), Group A obtained a $11 \%$ decrease in FP $(95 \%$ CI of $[-25,0.2])$ without statistical significance $(p=0.093)$ while Group B obtained a statistically significant $(p=0.036)$ increase of $19 \%$ in FP $(95 \%$ CI of $[16.5,28.8])$. When comparing groups, a statistical effect of the feedback method was observed on the evolution of FP rate after training $(p=0.009)$.

Improvement's difference in overall classification score doesn't seem to be due to a better classification of the retrained class (as shown in Fig. 5.B). Instead, this difference appears to be due to a decrease in False Positives for that class with the proposed feedback while Raw output feedback lead to an increase in False Positives (as shown in Fig. 5C).

2) Separability and overlapping between clusters of classes: The Figure 7 shows the averaged evolution of the separability between clusters measured by ratio $J_{3}$ for both groups. While the separability index were not statistically different between groups before the training ( $p=0.98)$, a significant $(p=0.003)$ difference in class separability variation can be seen with a $0.31 J_{3}$ mean-value for 
Group A (Pattern Similarity Biofeedback)

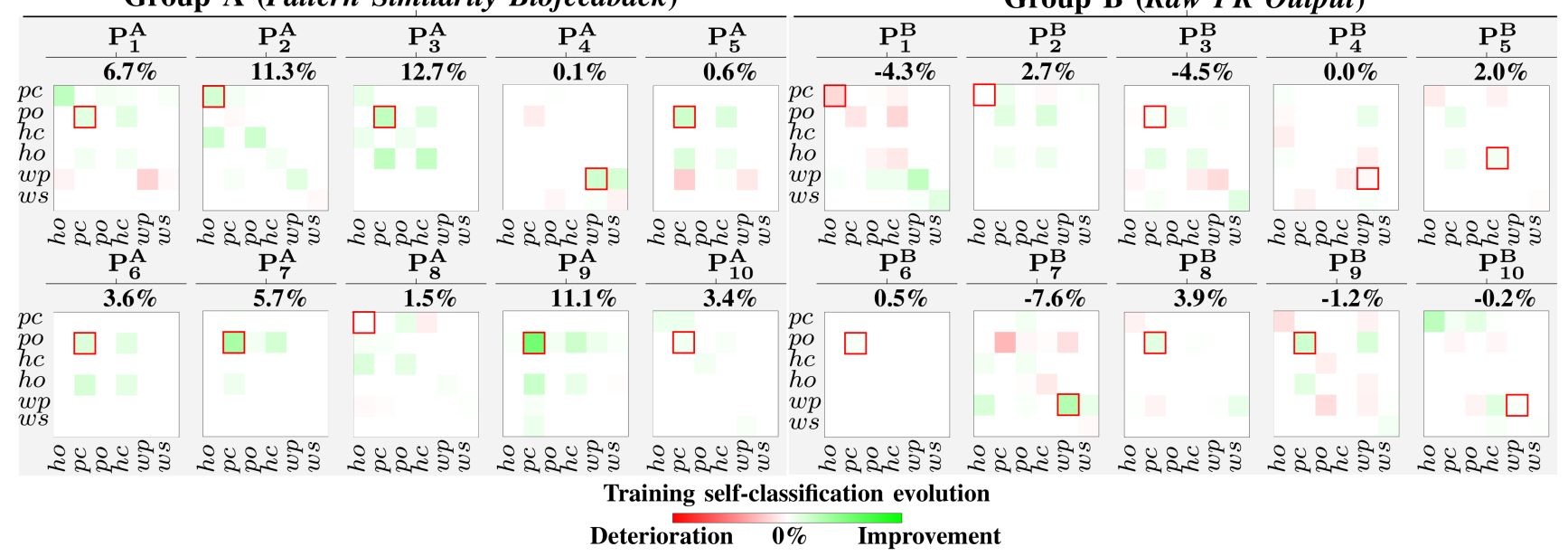

Fig. 6: Matrices representing the variation of the overlapping between clusters after the retraining of a class (marked with a red square) for each participant of the two groups. Decrease of overlapping is shown in non-diagonal cells with green background, while its increase is shown in non-diagonal cells with red background. Empty cells indicates the absence of variation after the retraining. See Appendix 1 for additional information on the definition of these matrices.

Group A and 0.06 for Group B. This indicates a clear overall effect of the proposed biofeedback on the class separability. Figure 6 shows the variations of the clusters

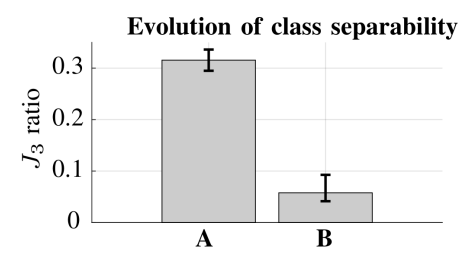

Fig. 7: Variation of averaged class-separability ratio index $\left(J_{3}\right)$ after training for the two groups $\mathrm{A}$ and $\mathrm{B}$, with representation (as error bars) of the $95 \%$ Confidence Interval.

overlapping matrices (computed as described in Appendix VII-A for each participants between before and after the training of user with feedback. Green background cells indicate an improvement, while red background cells show a deterioration. On the diagonal terms, green cells indicate an increase of the classification score after training, while red cells show its decrease. On the non-diagonal terms, green cells indicate a decrease of the overlapping space between given classes, while red cells show an increase of the overlapping space between given classes. Empty cells indicate a negligible variation (between $-5 \%$ and $+5 \%$ ) after the retraining. Considering all the movement classes and their associated clusters, after the training, clusters in Group A decrease on average their overlapping space with others of $5.7 \%$ with a $95 \%$ (C.I.) of $[5,6.5]$ while in group $\mathrm{B}$ it is decreased by $0.9 \%$ with a $95 \%$ (C.I.) of $[-1.9,1.7]$, with a statistical difference observed between the two groups ( $p=0.002)$. Considering only the cluster of the retrained movement (marked with a red square on the Fig. 6), its overlapped space with other clusters decreases of $22.9 \%$ with a $95 \%$ (C.I.) of $[16.9,23.6]$ for Group A, showing a statistical difference $(p=0.041)$ with Group B which presents an overlapping increase of $7.9 \%$ with a $95 \%$ (C.I.) of $[5.6,10.9]$. Indeed, all participants in Group A but $P_{4}^{A}$, $P_{5}^{A}$ and $P_{8}^{A}$ (which had already a initially well distributed space between clusters) have a better spatial organization of clusters after the retraining, with less overlapped clusters after the optimization. The absence of overlapping of the C.I. on the computed overlapping variations for the two groups indicating a clear effect of the feedback method. Looking at individual results in Group A, most of the participants (apart from $P_{8}^{A}$ ) decreased the overlapping of their retrained class (green cells in the diagonal) with, apart from participants $P_{1}^{A}, P_{4}^{A}$ and $P_{5}^{A}$, a decreased number of other movement cluster getting closer of another (reduced number of red cells in the non-diagonal cells). In Group B, no variation of overlapping for the retrained class were observed in half participants $\left(P_{2}^{B}, P_{4}^{B}, P_{5}^{B}, P_{6}^{B}, P_{10}^{B}\right)$. While the overlaps of the retrained cluster with other clusters did only clearly increased for 2 participants $\left(P_{1}^{B}\right.$ and $\left.P_{4}^{B}\right)$, the training had a visible negative effect (red cells outside of the diagonal) on other classes in almost all participants apart $P_{2}^{B}$.

3) Variations of muscles contraction strategies: Figure 9 presents with radar plots the average spatial repartition of muscle contractions (i.e. the averaged and normalized RMS amplitude on each sEMG channel) associated to the retrained movement class, before and after training, for each participant.

Comparing the shapes of associated octagons, the muscle contraction patterns in Group A appears changed rather importantly after retraining (especially for $P_{2}^{A}, P_{4}^{A}, P_{5}^{A}$, $P_{7}^{A}, P_{8}^{A}$ and $P_{9}^{A}$ ), compared to Group B in which a muscle contraction scaling effect (rather than a shape variation) can be observed in most participants (except $P_{2}^{B}, P_{4}^{B}$ and $P_{10}^{B}$ ) thus indicating a minimal variation of activation patterns and associated motor strategies. 


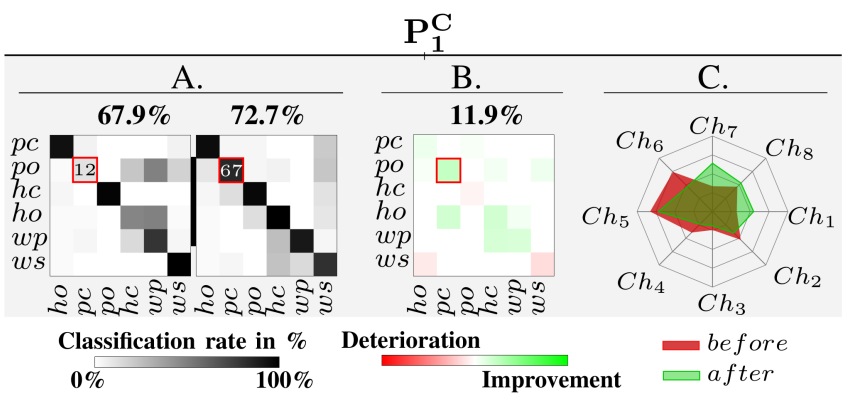

Fig. 8: Results of the amputated participant. A: Confusion matrices before and after training with the Pattern Similarity Biofeedback (the percentage over each matrix indicates the average global classification score). B: Variation of the overlapping between clusters after the retraining of a class (marked with a red square). C: average muscular contraction (normalized RMS of sEMG signals) pattern for the retrained movement, before and after training.

\section{B. Amputated participant}

Figure 8 A shows that, when considering all the movement's classes, the participant obtained an average increase in classification score of respectively $4.8 \%$ after the retraining with the pattern similarity biofeedback. Nonetheless, looking only at the "retrained" pinch opening class (marked with a red square on Fig. 8 A), the use of training with proposed biofeedback allowed an increase of $55 \%$ in the recognition rate. False positive for class $\mathrm{PO}$ did not vary significantly since its rate increased by only $4 \%$ after training.

Considering the variation of overlapping between clusters of classes shown in Fig. 8.B, it can be seen that the pinch opening cluster tend to move away from other clusters with a $11.9 \%$ reduction in overlapping. As shown in Fig. 8 C, the participant appears to have found a novel optimal muscular contraction strategy to perform the pinch opening, which is rather different from the one initially used.

\section{DISCUSSION}

\section{A. The need of improved biofeedback to train users}

Mastering precise myoelectric control through multielectrodes and PR control approaches remains difficult to master for amputated participants [4]. Users of those systems have to go through rather complex training and learning processes. With the recent availability of more active DoF and associated PR control approaches in commercial products (e.g., the COAPT ${ }^{\circledR}$ system, http://www.coaptengineering.com/, or the recent OttoBock Myo Plus ${ }^{\circledR}$, https://www.ottobock.com/), there is definitively a growing need for adapted tools and protocols to train users in a simple and efficient way, particularly in clinical environments with medical staff which are not machine learning experts and have a limited ability to master the tuning of those technical tools. It has been shown that user's learning step could generally happen without any external feedback, yet with extended training phases [22]. More recently, ablebodied participants were shown to be able to train and improve their PR control without coaching or external feedback, just relying on inner focus [26]. Nonetheless, several studies illustrated the possible enhancement permitted on the learning ability and the reduction of learning time when adequate external feedback is provided [25], [28], whether it is brought by a visual feedback or by the experimenters. Similarly, [26] showed that, in able-bodied participants, the absence of feedback did affect the quality of the EMG patterns by leading to the exhibition of higher amplitude patterns in participants.

One major challenge with these PR control algorithms generally appearing as complex "black boxes"- is to provide simple, understandable and intuitive information allowing user to close their sensorimotor control loop to be able to learn in a faster and easier way the use of PR control and adapt their motor behavior accordingly.

\section{B. Effect on classification performance in able-bodied par- ticipants}

We here proposed a simple pattern similarity biofeedback which is intended to help users improving the distinguishability of the muscle activity pattern associated to a movement class with recognition issues, without affecting the overall classification performance (i.e. the successful recognition of other patterns) through over-training. The results of the experimental sessions provides a preliminary demonstration of the possibilities offered by this approach.

Experiments on naive able-bodied participants retraining only one problematic movement class, showed that the proposed biofeedback allowed an overall classification improvement of $10.2 \%$ compared to the $0.2 \%$ obtained with conventional training based on raw PR output feedback. This last method, while leading to similar improvement of the recognition score of the retrained class $(40.4 \%$ vs $39.4 \%$ for the proposed method), tends to have a limited or negative impact on the overall PR recognition score (as seen on Fig 5 , due to in increase in false positives for the retrained class.

The improvement obtained in overall classification score $(10.5 \%)$, is smaller than the one of $23 \%$ obtained by [26] after 30-minutes training sessions during 5 days, but is promising since it was obtained after a single 3 minutes session. Similarly to [28], we observed that providing continuous feedback on classifier's clustering map rather than a raw output (class label) allows an accelerated increase in classification performance, with a rather similar evolution of the scores.

Similarly, [25] obtained an important increase of $16.9 \%$ of classification score, but after a 2-weeks training period (with a session duration over 1 hour). Extending session duration and number of repetitions could possibly lead to even higher improvements with our method, even if the goal of our objective is rather to make the participant change his/her motor behavior rather than refining it through extended training. Nonetheless the limited duration of the training in our experiment is probably not sufficient for participants to keep in mind the new optimized gesture (discovered during exploration with feedback) for a long period of time. 
Group A (Pattern Similarity Biofeedback)

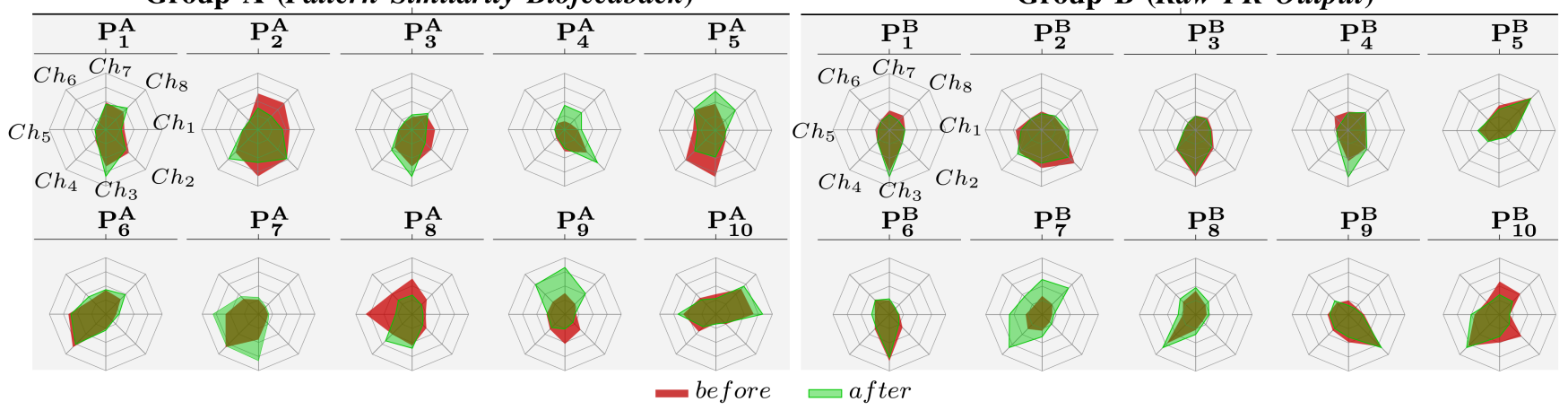

Fig. 9: Average muscular contraction (RMS of sEMG signals, normalized for each participant according to the maximum RMS value measured during first training) patterns for the retrained movement, before and after training, for each participant.

The regular repetition of the protocol would probably be necessary, at least for this novel optimal motor strategy to become natural and automatic to the participants.

In conclusion, similarly to [27], we observed that a structured training during one single session could improve the myoelectric control of a multi-functional device. Nonetheless, we show here that visual feedback can be used to drive users in finding more decodable muscle contraction strategies rather than making them better in using a predefined fixed strategy.

\section{Guiding users toward different motor strategies}

The analysis of the separability and variations of the overlapping between clusters after the retraining of the classes (see Fig. 7 and Fig. 6 6 indeed showed that the proposed method allowed users to find new ways in performing muscular patterns which improves the distinguishability of patterns (i.e. the inter-class separability and overlapping) rather than just improving user's ability to repeat a given pattern used to train the PR algorithm. The changes in the spatial distribution of muscular activities after the user training step (as shown in Fig. 9p, which are more important for the participants trained with our pattern similarity biofeedback, support this hypothesis. Participants were more likely to find new and different muscle contraction strategies when feedback provided information on the distance to other patterns of the PR training set (PSB-group) rather than information on the similarity with those (RF-group). It is also important to highlight the fact that this improvement was achieved without the need of a PR expert guiding the user, without providing any precise and complex explanation of the overall PR principles, nor without the participants having to decode information from complex and rich visual feedback.

\section{Experiments on amputated participant}

Preliminary experiments on oneamputee experienced with myoelectric control showed more modest improvements. Nonetheless it allowed him to find a novel way of "opening the pinch" with his phantom limb which improved its recognition by the classifier, and this without the help or instruction of the experimenter. Thus, such approach could be particularly useful for amputated participant which may have sometimes trouble providing clear information about the nature of the phantom limb mobilization or muscular pattern contraction they are exhibiting, which in return makes it hard for an, even skilled, experimenter to provide hints on strategy modification to improve the overall performance. We also believe that such method could make an even bigger difference in larger sets of classes (including individual finger movements for example) in which there are more chances to have overlapping between classes.

\section{E. Study limitations and future work}

This work is a preliminary demonstration that the proposed feedback might be useful, but the experimental protocol in this study remains far from the envisioned clinical application. Thus a more clinically relevant protocol involving more participants, in longer training protocols, possibly with multiple classes training (one after the other) and a repetition of training sessions across days or weeks would be necessary to study stability of improvement and ensure that the novel muscular strategies are sustainably learned and not only a temporary motor adaptation. Experimental comparison with other existing training protocols (without feedback, or for example, with EMG radar plots as in [27]) would be necessary, also to distinguish effect of feedback from training time. Our results were also obtained in a controlled environment with limited variations of arm posture, muscle fatigue, or more variations in the contractions force levels or timings, which are known to be perturbing parameters in PR. Robustness of PR is fundamental and thus performing evaluations in a perturbed environment would be necessary to fully appreciate the possibility of the proposed method. Finally, a statistical analysis to determine possible influencing factors (like the retrained class, the age of participants or their amount of experience with myoelectric control, etc.) will be the subject of a future work. While we principally focused on the adaptation of user behavior, alternative approaches, which rather focus on the continuous adaptation and optimization of the prosthetic control policies but directly driven by simple feedback from user (discrete reward), through the user of reinforcement-based machine learning framework 
as described in [31] could also contribute to additional personalization and control performance increase.

\section{CONCLUSIONS}

An intuitive visual biofeedback dedicated to train optimally participants in using myoelectric PR based control approaches was proposed. Such tool used during user's training phases allows them to learn to adapt and modulate their muscular contraction strategies to improve the distinguishability of their muscular patterns. Experiments conducted on a group of able-bodied participants and one amputee confirmed the potential of the proposed approach compared to generic feedback, allowing (in a reduced amount of training time) an increase of the classification score for a given problematic class without impacting the others or affecting the overall classification performance. These first encouraging results clearly highlight the potential of such online biofeedback method which does not focus so much on extensively training users than on providing them with information to adapt or even radically change their motor strategies to solve some PR misclassification issues.

Future work will be focused on conducting extended experimental protocols with more amputated participants in a more realistic and thus perturbed environment, to clearly evaluate the real possibilities of our pattern similarity biofeedback.

\section{APPENDIX}

A. Metrics to characterize the variation of overlapping in clusters distribution

This metric allows to compare the clusters distribution between the different LDA projected spaces created in steps 1 and 3 (PR training before and after subject's training with feedback). Basically, this metrics evaluates with generic confusion matrices how good is the training to classify itself, i.e. testing the PR algorithm capacity to classify the same training data used to initially train it. Practically, a LDA space is first created using training data. Then, each point of this same training dataset is classified using the LDA classifier. Such classification should lead to a perfect classification score if the training data are not overlapped in the projected LDA space. Similarly, all the non diagonal terms (misclassification points) appearing on the obtained confusion matrices will indicate the existence of overlapping between classes clusters in the LDA space. Such confusion matrices are thus first created separately for step 1 and step 3 . Then, the variation between the two confusion matrices is plotted to illustrate the evolution of overlapping between the two steps, as shown in Fig. 10 .

A specific color code is used to ease the reading on this generated matrix: green color indicates an improvement while red color a deterioration. More precisely, on the diagonal terms, green cells indicate an increase after training of the classification score for the given class, while red cells indicate a decrease after training of the classification score. On the non-diagonal terms, green cells indicate a decrease of the overlapping space between given classes corresponding

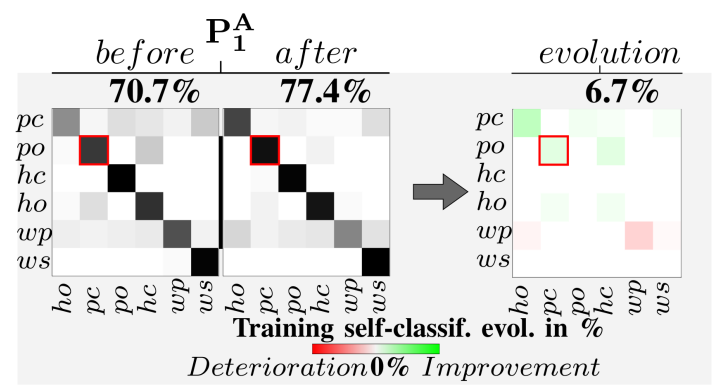

Fig. 10: Confusion training matrices of step 1 and 3 for participant $P_{1}$ of group A, along with the matrix representing the variation of the overlapping between clusters after the retraining of a class (marked with a red square).

to a positive evolution. Red cells indicate an increase of the overlapping space between given classes corresponding to a negative evolution. Empty cells indicate a negligible variation (between $-5 \%$ and $+5 \%$ ) after the retraining.

\section{ACKNOWLEDGMENT}

The authors warmly thank the participants included in this protocol, and Yvonne Janssen and Jozina de Graaf for their help with the statistical analysis. The study was financially supported by the ANR (project PhantoMovControl ANR-15CE19-0008-02).

\section{REFERENCES}

[1] E. D. Sherman, "A russian bioelectric-controlled prosthesis: Report of a research team from the rehabilitation institute of montreal," Canadian Medical Association Journal, vol. 91, no. 24, p. 1268, 1964.

[2] L. Resnik, S. L. Klinger, and K. Etter, "The deka arm: Its features, functionality, and evolution during the veterans affairs study to optimize the deka arm," Prosthetics and orthotics international, vol. 38, no. 6, pp. 492-504, 2014.

[3] J. T. Belter and A. M. Dollar, "Performance characteristics of anthropomorphic prosthetic hands," in Rehabilitation Robotics (ICORR), 2011 IEEE Int. Conf. on, pp. 1-7, 2011.

[4] C. Castellini, P. Artemiadis, M. Wininger, A. Ajoudani, M. Alimusaj, A. Bicchi, B. Caputo, W. Craelius, S. Dosen, K. Englehart, et al., "Proceedings of the first workshop on peripheral machine interfaces: going beyond traditional surface electromyography," Frontiers in neurorobotics, vol. 8, 2014.

[5] F. R. Finley and R. W. Wirta, "Myocoder studies of multiple myopotential response.," Archives of physical medicine and rehabilitation, vol. 48, no. 11, p. 598, 1967.

[6] P. Herberts, C. Almström, R. Kadefors, and P. D. Lawrence, "Hand prosthesis control via myoelectric patterns," Acta Orthopaedica Scandinavica, vol. 44, no. 4-5, pp. 389-409, 1973.

[7] P. Lawrence, P. Herberts, and R. Kadefors, "Experiences with a multifunctional hand prosthesis controlled by myoelectric patterns," Advances in External Control of Human Extremities, pp. 47-65, 1973.

[8] A. Al-Timemy, G. Bugmann, J. Escudero, and N. Outram, "Classification of finger movements for the dexterous hand prosthesis control with surface electromyography," Biomedical and Health Informatics, IEEE J. of, vol. 17, no. 3, pp. 608-618, 2013.

[9] D. Farina, N. Jiang, H. Rehbaum, A. Holobar, B. Graimann, H. Dietl, and O. Aszmann, "The extraction of neural information from the surface emg for the control of upper-limb prostheses: emerging avenues and challenges," Neural Systems and Rehabilitation Engineering, IEEE Trans. on, vol. 22, no. 4, pp. 797-809, 2014.

[10] E. Scheme and K. Englehart, "Electromyogram pattern recognition for control of powered upper-limb prostheses: state of the art and challenges for clinical use.," J. of Rehabilitation Research \& Development, vol. 48 , no. $6,2011$. 
[11] S. Muceli, N. Jiang, and D. Farina, "Extracting signals robust to electrode number and shift for online simultaneous and proportional myoelectric control by factorization algorithms," Neural Systems and Rehabilitation Engineering, IEEE Trans. on, vol. 22, no. 3, pp. 623633, 2014.

[12] J. He and X. Zhu, "Combining improved gray-level co-occurrence matrix with high density grid for myoelectric control robustness to electrode shift," Neural Systems and Rehabilitation Engineering, IEEE Trans. on, vol. 25, no. 9, pp. 1539-1548, 2017.

[13] M. Ortiz-Catalan, B. Håkansson, and R. Brånemark, "An osseointegrated human-machine gateway for long-term sensory feedback and motor control of artificial limbs," Science translational medicine, vol. 6, no. 257, pp. 257re6-257re6, 2014.

[14] E. Mastinu, P. Doguet, Y. Botquin, B. Håkansson, and M. OrtizCatalan, "Embedded system for prosthetic control using implanted neuromuscular interfaces accessed via an osseointegrated implant," Biomedical Circuits and Systems, IEEE Trans. on, vol. 11, no. 4, pp. 867-877, 2017.

[15] C. Lauretti, A. Davalli, R. Sacchetti, E. Guglielmelli, and L. Zollo, "Fusion of m-imu and emg signals for the control of trans-humeral prostheses," in Biomedical Robotics and Biomechatronics (BioRob), 2016 6th IEEE Int. Conf. on, pp. 1123-1128, 2016.

[16] D. M. Wolpert, Z. Ghahramani, and J. R. Flanagan, "Perspectives and problems in motor learning," Trends in cognitive sciences, vol. 5, no. 11, pp. 487-494, 2001.

[17] G. Nakamura, T. Shibanoki, Y. Kurita, Y. Honda, A. Masuda, F. Mizobe, T. Chin, and T. Tsuji, "A virtual myoelectric prosthesis training system capable of providing instructions on hand operations," Int. J. of Advanced Robotic Systems, vol. 14, no. 5, 2017.

[18] F. Anderson and W. F. Bischof, "Augmented reality improves myoelectric prosthesis training," Int. J. on Disability and Human Development, vol. 13, no. 3, pp. 349-354, 2014.

[19] B. D. Winslow, M. Ruble, and Z. Huber, "Mobile, game-based training for myoelectric prosthesis control," Frontiers in bioengineering and biotechnology, vol. 6, p. 94, 2018.

[20] A. L. Fougner, Ø. Stavdahl, and P. J. Kyberd, "System training and assessment in simultaneous proportional myoelectric prosthesis control," J. of neuroengineering and rehabilitation, vol. 11, no. 1, p. $75,2014$.

[21] S. Dosen, M. Markovic, K. Somer, B. Graimann, and D. Farina, "Emg biofeedback for online predictive control of grasping force in a myoelectric prosthesis," J. of neuroengineering and rehabilitation, vol. 12, no. 1, p. 55, 2015.

[22] J. He, D. Zhang, N. Jiang, X. Sheng, D. Farina, and X. Zhu, "User adaptation in long-term, open-loop myoelectric training: implications for emg pattern recognition in prosthesis control," $J$. of neural engineering, vol. 12, no. 4, p. 046005, 2015.

[23] J. M. Hahne, S. Dähne, H.-J. Hwang, K.-R. Müller, and L. C. Parra, "Concurrent adaptation of human and machine improves simultaneous and proportional myoelectric control," Neural Systems and Rehabilitation Engineering, IEEE Trans. on, vol. 23, no. 4, pp. 618-627, 2015.

[24] E. Scheme and K. Englehart, "Training strategies for mitigating the effect of proportional control on classification in pattern recognition based myoelectric control," J. of prosthetics and orthotics: JPO, vol. 25, no. 2, p. 76, 2013.

[25] M. Powell, R. Kaliki, and N. Thakor, "User training for pattern recognition-based myoelectric prostheses: improving phantom limb movement consistency and distinguishability," Neural Systems and Rehabilitation Engineering, IEEE Trans. on, vol. 22, no. 3, pp. 522532, 2014.

[26] M. B. Kristoffersen, A. W. Franzke, C. K. van der Sluis, A. Murgia, and R. M. Bongers, "The effect of feedback during training sessions on learning pattern-recognition-based prosthesis control," Neural Systems and Rehabilitation Engineering, IEEE Trans. on, vol. 27, no. 10, pp. 2087-2096, 2019.

[27] A. D. Roche, I. Vujaklija, S. Amsüss, A. Sturma, P. Göbel, D. Farina, and O. C. Aszmann, "A structured rehabilitation protocol for improved multifunctional prosthetic control: a case study," JoVE (J. of Visualized Experiments), no. 105, p. e52968, 2015.

[28] Y. Fang, D. Zhou, K. Li, and H. Liu, "Interface prostheses with classifier-feedback-based user training," Biomedical Engineering, IEEE Trans. on, vol. 64, no. 11, pp. 2575-2583, 2017.

[29] K. Englehart, B. Hudgins, P. Parker, and M. Stevenson, "Classification of the myoelectric signal using time-frequency based representations," Medical engineering \& physics, vol. 21, no. 6, pp. 431-438, 1999.
[30] R. O. Duda, P. E. Hart, and D. G. Stork, Pattern classification. John Wiley \& Sons, 2012.

[31] P. M. Pilarski, M. R. Dawson, T. Degris, F. Fahimi, J. P. Carey, and R. S. Sutton, "Online human training of a myoelectric prosthesis controller via actor-critic reinforcement learning," in 2011 IEEE Int. Conf. on rehabilitation robotics, pp. 1-7, 2011.

[32] M. Oskoei and H. Hu, "Support vector machine-based classification scheme for myoelectric control applied to upper limb," Biomedical Engineering, IEEE Trans. on, vol. 55, no. 8, pp. 1956-1965, 2008.

[33] M. Zardoshti-Kermani, B. Wheeler, K. Badie, and R. Hashemi, "Emg feature evaluation for movement control of upper extremity prostheses," Rehabilitation Engineering, IEEE Trans. on, vol. 3, no. 4, pp. 324-333, 1995.

[34] D. Tkach, H. Huang, and T. Kuiken, "Research study of stability of time-domain features for electromyographic pattern recognition," J Neuroeng Rehabil, vol. 7, p. 21, 2010.

[35] S. Theodoridis and K. Koutroumbas, Pattern recognition. Elsevier, 2003. 